\title{
High-resolution spectral domain-optical coherence tomography in multiple sclerosis, Part II - the total macular volume. The first follow-up study over 2 years
}

\section{Nermin Serbecic ${ }^{1,2}{ }^{*}{ }^{\dagger}$, Fahmy Aboul-Enein ${ }^{3 \dagger}$, Sven C. Beutelspacher ${ }^{2}$, Adnan Khan ${ }^{4}$, Clemens Vass $^{1}$, Wolfgang Kristoferitsch ${ }^{3}$, Andreas Reitner ${ }^{1}$ and Ursula Schmidt-Erfurth ${ }^{1}$}

1 Department of Ophthalmology, Medical University of Vienna, Vienna, Austria

2 Department of Ophthalmology, Faculty of Medicine Mannheim, University of Heidelberg, Mannheim, Germany

${ }^{3}$ Department of Neurology, SMZ-Ost Donauspital, Vienna, Austria

${ }^{4}$ Nuffield Department of Surgical Sciences, Division of Medical Sciences, University of Oxford, Oxford, UK

Edited by:

Kenneth Shindler, University of

Pennsylvania, USA

\section{Reviewed by:}

Benjamin Osborne, Georgetown

University Hospital, USA

Laura Balcer, New York University

School of Medicine, USA

*Correspondence:

Nermin Serbecic, Department of Ophthalmology, Faculty of Medicine

Mannheim, University of Heidelberg

Mannheim, Germany

e-mail: nermin.serbecic@medma.

uni-heidelberg.de

${ }^{\dagger}$ Nermin Serbecic and Fahmy Aboul-Enein have contributed equally to this work.
Background: Recent studies investigating the use of optical coherence tomography (OCT) in multiple sclerosis (MS) patients have resulted in wide-ranging and often contradictory outcomes. This is mainly due to the complex etiology and heterogeneity of MS, physiological variations in the retinal nerve fiber layer (RNFL) and/or total macular volume (TMV), and limitations in methodology. It remains to be discovered whether any retinal changes in MS develop continuously or in a stepwise fashion, and whether these changes occur in all or a subset of patients. High-resolution spectral domain-OCT devices (SD-OCT) would be required to detect subtle retinal changes and longitudinal studies would have to be carried out to investigate retinal changes over time. In addition, if the hypothesis is correct, then retinal and global brain tissue changes should be detected in a substantial majority of MS patients and detection should be possible with a high degree of disease activity and/or long disease course.

Methodology: In order to address the factors above, $37 \mathrm{MS}$ patients (relapsing-remitting, $n=27$; secondary progressive, $n=10$ ) were examined prospectively on two occasions with a median interval of $22.4 \pm 0.5$ months [range 19-27]. SD-OCT was utilized with the Spectralis $3.5 \mathrm{~mm}$ circle scan protocol (with locked reference images and eye-tracking mode). None of the patients had optic neuritis 12 months prior to study entry or during the observation period.

Principal Findings: The initial TMV pattern differed between study participants, but remained relatively unchanged over the 2-year observation period despite high disease activity or long disease course. The TMV correlated well with the RNFL.

Conclusion: The significance of differences in TMV (and RNFL) between study participants remains unclear. Until these differences have been explored further, OCT data in MS patients should be interpreted with caution.

Keywords: MS, OCT, macula lutea, neurodegenerative diseases, MRI

\section{INTRODUCTION}

It has recently been described that the retinal nerve fiber layer (RNFL) thickness in multiple sclerosis (MS) patients can be reliably visualized by optical coherence tomography (OCT), and that RNFL reduction correlates to diffuse axonal and/or neuronal degeneration throughout the CNS. This is regardless of whether the eyes have previously been affected by optic neuritis (ON). In addition to the ganglion cell and RNFLs, the total macular volume (TMV) has also been described as a reliable variable in the use of OCT in MS patients.

Loss of TMV was described as an additional neuromarker for the degree of global degeneration of myelin, axons, oligodendrocytes, and astrocytes throughout the CNS of MS patients.
Furthermore, changes in TMV were suggested to be more reliable at detecting global neurodegeneration than the observed decrease in the RNFL thickness (1-3).

However, some crucial questions still remain:

(1) How and why would 2.5 million axons of both optic nerves be representative of billions of axons within the entire CNS?

(2) Is there a continuous, degenerative process in the entire CNS taking place in every MS patient, and if so, does it proceed at the same pace in all CNS areas simultaneously? How does this hypothesis apply to those MS patients who follow a clear relapsing-remitting disease course with only few relapses and who are not disabled over decades (4)? 
(3) The term "neurodegeneration," when applied to MS, needs a clear definition. What is meant by primary or secondary degeneration of neurons? Neuropathologists still classify MS as a white matter disease, although it has been established for more than a century that myelin, oligodendrocytes, astroglia, and axons are damaged in MS lesions, and that white and gray matter are both affected (5). It seems that, in recent years, there has been significant distortion in semantics, which has impeded uniformity between studies (ranging from the use of axonal damage over degeneration of axons, or moving from neuronal degeneration to "neurodegeneration"). In any case, the term "neurodegeneration" implies a primary degenerative process of neurons, and thus should be avoided as long as direct scientific evidence is lacking.

(4) Can the hypothesized retinal changes described above be detected with every OCT device, or is there a minimal technical standard required, as the latest high-resolution SD-OCT is currently assumed to be the golden standard. $(1,6-8)$ ?

(5) Can very subtle changes, described above, be separated from gross changes caused by $\mathrm{ON}$ or even physiological variations $(6-8)$ ?

(6) Are OCT devices really suitable for the detection and monitoring of expected, subtle RNFL- and TMV changes as there is yet no reliable and normative database available for this specific indication $(6,8)$.

Most importantly, the existing literature and prospective reports utilizing SD-OCT in MS studies must be interpreted with caution, as even physiological variations of the RNFL or TMV (in addition to the hypothesized subtle retinal changes of $<2-4 \mu \mathrm{m}$ /year) were found to exceed the detection-threshold of high-resolution spectral domain-OCT (SD-OCT) devices (8). To date, most of the OCT studies were performed with conventional time-domain OCT, which has proved to be far inferior to highresolution SD-OCT, lacking the ability to measure changes in RNFLT of identical retinal locations over time (9).

To address the factors described and avoid methodological limitations, we utilized a high-resolution SD-OCT and a well-classified cohort of 27 RRMS and 10 SPMS patients with partly intense disease activity and high relapse-rates over a prolonged observation period of approximately $22.4 \pm 0.5$ months.

\section{MATERIALS AND METHODS \\ PARTICIPANTS}

This study was approved by the local Ethics Committee (Commission of Medical Ethics of Vienna; Ethic Approval/Registration Number: EK-08-028-0308 and Ethical Commission of the Medical University of Vienna; Ethic Approval/Registration Number: 414/2008). Informed written consent was obtained from all patients and volunteers before study entry.

We recently reported both the demographic and clinical data, the RNFL baseline characteristics of a total of 59 MS (42 RRMS, 17 SPMS) patients, and the follow-up RNFL characteristics of those 37 MS patients (27 RRMS, 10 SPMS) who were willing to participate further $(6,8)$. The TMV was determined in each case in the same session as the RNFL. Patients were encouraged to drink sufficiently (1-2 L) 1-2 h before each session to guarantee hydration.

In each case, the diagnosis of MS was based on a combination of clinical course, MRI, cerebrospinal fluid (CSF) analysis, and the exclusion of other disorders or diseases (10-12). Oligoclonal bands were found in the CSF samples of all MS patients. Patients with other diseases that reduce RNFL thickness such as glaucoma, anterior ischemic optic neuropathy, high myopia, and congenital abnormalities of the optic nerves were excluded from the study.

Baseline clinical neurological examinations, visual evoked potentials (VEP), and ophthalmologic examinations were performed within 7 days. None of the patients had a history of ON within 12 months prior to the onset of the study. A summary of detailed demographic and clinical data for each patient is given in Table 1 (8).

\section{HIGH-RESOLUTION SPECTRAL DOMAIN-OCT}

We used a high-resolution SD-OCT, which combines OCT technology with a confocal scanning laser ophthalmoscope (Heidelberg Engineering, Heidelberg, Germany, Spectralis software version 4.0.3.0, Eye Explorer Software 1.6.1.0, in addition to Eye Explorer software version 1.6.2.0 due to changes by the manufacturer during the follow-up period). A special eye-tracking mode (TrueTrac ${ }^{\mathrm{TM}}$ ) combined with a high scanning speed $(40,000$ A-scans/s) with an axial resolution of $7 \mu \mathrm{m}$ and a transversal resolution of $14 \mu \mathrm{m}$ allows for the reduction of artifacts due to eye movement. Each macular OCT scan is registered and locked to a reference infrared image. In addition, an automatic real-time averaging mode (ART mode) allows for adjustment of the recorded frames to obtain averaged B-scans, which enhances image quality by optimizing the signal-to-noise ratio. The eye tracker and automatic real-time averaging modes of the Spectralis SD-OCT system were used throughout the study. For follow-up scanning, the OCT software could identify previous scan locations and "guide" the OCT laser beam to scan the same location repeatedly. All automated measurements of macular thickness and volume were performed through dilated pupils with a high-resolution macular scan protocol allowing for a more detailed differentiation of retinal layers. (The TMV compounds of inner limiting membrane, nerve fiber layer, ganglion cell layer, inner plexiform layer, inner nuclear layer, outer plexiform layer, outer nuclear layer, external limiting membrane, photoreceptor layer, and retinal pigment epithelium.)

All macular scans were performed by one skilled and trained observer (NS) within one session and repeated if necessary, until a high-quality macular scan was achieved for use in further analysis. Final analysis was only performed on scans without segmentation errors and no manual correction was performed in any case. The observer had no knowledge of any clinical data or the specific baseline data. The SD-OCT imaging protocol comprised 49 B-scans per volume scan of $20^{\circ} \times 20^{\circ}$, and each scan was averaged with 9 frames per B-scan. Topographic macular surface maps were constructed automatically by the OCT software and displayed with numeric averages of the mean thickness for each of the nine map sectors (F, foveal; TI, inner temporal; TO, outer temporal; II, inner inferior; IO, outer inferior; NI, inner nasal; NO, outer nasal; SI, inner superior; $\mathrm{SO}$, outer superior) within three concentric regions 
Table 1 | Summary of demographic and clinical data.

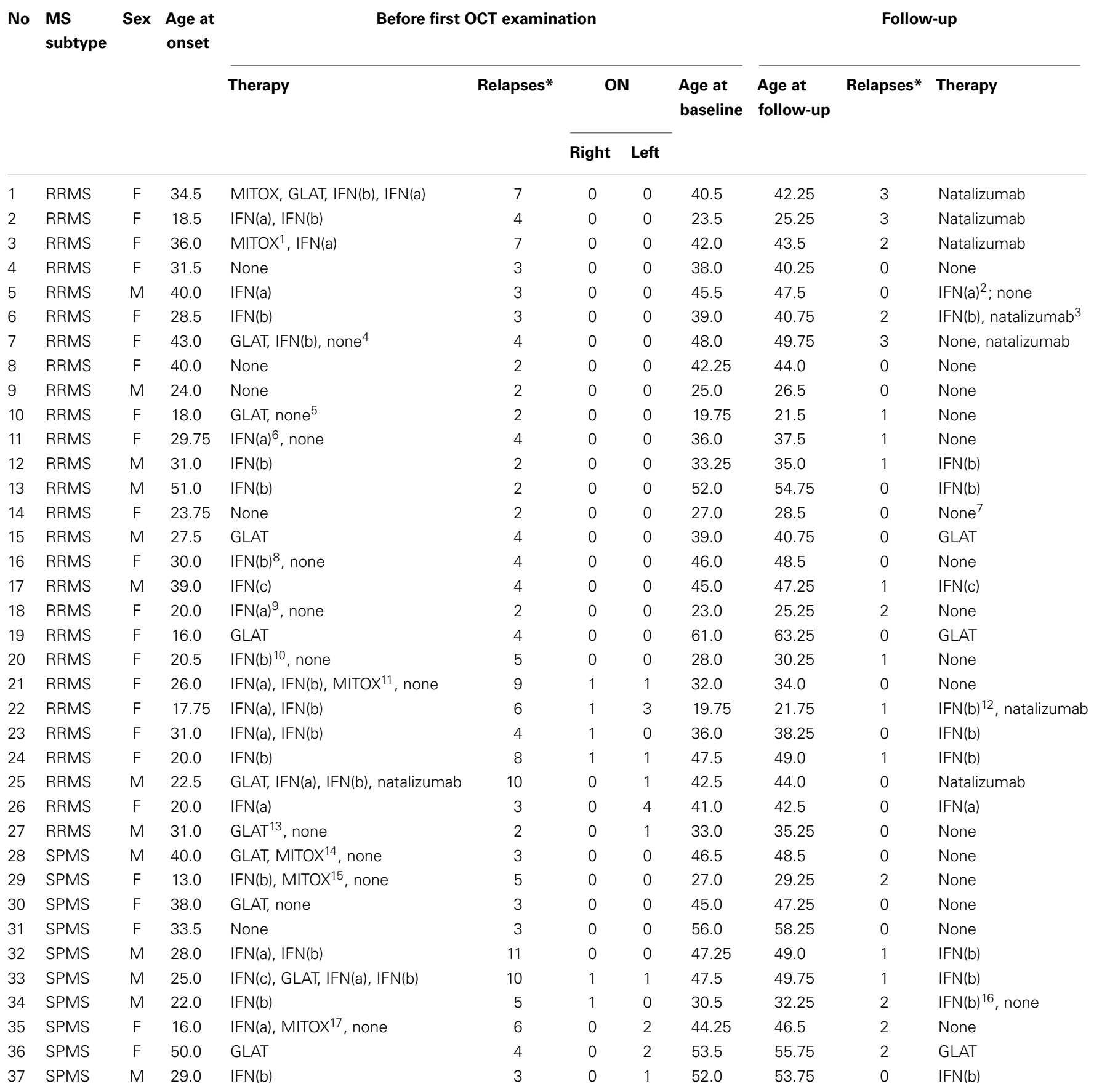

ON, optic neuritis; *, relapses treated with high dose steroid pulse therapy; no included patient had an ON within 12 months prior to the beginning of the study; GLAT, glatiramer-acetate $20 \mathrm{mg}$ subcutaneous once daily; MITOX, mitoxantrone; IFN(a), interferon beta 1a intramuscularly once/week; IFN(b), interferon beta 1a $(44 \mu \mathrm{g})$ subcutaneous trice/week; IFN(c), interferon beta $1 \mathrm{~b}(250 \mu \mathrm{g})$ subcutaneous alternate day; 1 , discontinued $48 \mathrm{mg}$ mitoxantrone/ $\mathrm{m}^{2}$ body surface); none, neither specific immunomodulatory or immunosuppressive therapy, drug holiday; 2, drug withdrawal 9 months after first OCT examination; 3, start 7 months before second OCT examination; 4, drug withdrawal 12 months before first OCT examination; 5, drug withdrawal 6 months before first OCT examination; 6, drug withdrawal 20 months before first OCT examination; 7, strict vegan diet caused vitamin B12 and folate deficiency; 8, high titers of anti-interferon autoantibodies, drug withdrawal 14 months before first OCT examination; 9, drug withdrawal 25 months before first OCT examination; 10, drug withdrawal 8 months before first OCT examination; 11 , mitoxantrone cumulative dose $96 \mathrm{mg} / \mathrm{m}^{2}$ body surface, drug withdrawal 10 months before first OCT examination; 12 , change to natalizumab 2 months after first OCT examination; 13, drug withdrawal 6 months before first OCT examination; 14, mitoxantrone cumulative dose $92 \mathrm{mg} / \mathrm{m}^{2}$ body surface, drug withdrawal $10 \mathrm{months}$ before first OCT examination; 15, mitoxantrone cumulative dose $92 \mathrm{mg} / \mathrm{m}^{2}$ body surface, drug withdrawal 26 months before first OCT examination; 16, drug withdrawal 5 months after first OCT examination 17, mitoxantrone cumulative dose $108 \mathrm{mg} / \mathrm{m}^{2}$ body surface, drug withdrawal 27 months before first OCT examination. Table 1 was already published in Ref. (7). 
of 1, 3, and $6 \mathrm{~mm}$ diameter, respectively, as defined by the Early Treatment Diabetic Retinopathy Study (ETDRS).

\section{VISUAL FUNCTION TESTING, VISUAL FIELD ANALYSIS, VISUAL EVOKED POTENTIALS}

Visual function testing, visual field analysis, and VEP have been described previously $(6,7)$.

\section{STATISTICS}

The statistics used in this study has been described previously $(6-8)$.

\section{RESULTS}

The results of our study performed with the high-resolution SDOCT can be summarized briefly as follows: over a median observation period of $22.4 \pm 0.5$ months (range from 19 to 27 months), the TMV measurements of each MS patient were unchanged compared to baseline (Figure 1). The minimal positive/negative changes seen in some follow-up scans were within the intersession/observer variation (Figures 1 and 2) (3, 6-8). Mean TMV values were found highest in RRMS patients without ON. Mean TMV values were found lower in RRMS with ON and in SPMS with and without ON (Figure 2). The visual and contrast acuity and sensitivity tests (ETDRS, Sloan and Pelli-Robson-charts), the color vision test (Lanthony D-15), the Humphrey visual field analysis, and the VEP also showed no changes compared to baseline values (8). Again, neither age, disease duration, or MS subtype was found to correlate with TMV changes, but TMV values were found moderately correlated to the RNFL values (Figure 3).

\section{DISCUSSION}

A number of cross-sectional OCT-trials have demonstrated a decrease in the RNFLT of patients with MS compared with healthy controls, which was more prominent in progressive disease than in the RR course (13-15). This has led to the assumption that the decrease of RNFLT may reflect "neurodegeneration," cerebral atrophy, and a progressive disease course $(13,15)$. This has yet to be confirmed in prospective, controlled longitudinal studies (16). Loss of TMV has been considered as an even more reliable marker for "neurodegeneration" (2).

In this long-term follow-up trial, we were not able to detect any significant reduction of TMV in MS patients over an observation period of $22.4 \pm 0.5$ months [range 19-27]. All TMV variations ( \pm compared to baseline) were within the intersession and measurement variability of the high-resolution SD-OCT device used in the study (17). The results of our study reflect the negative data

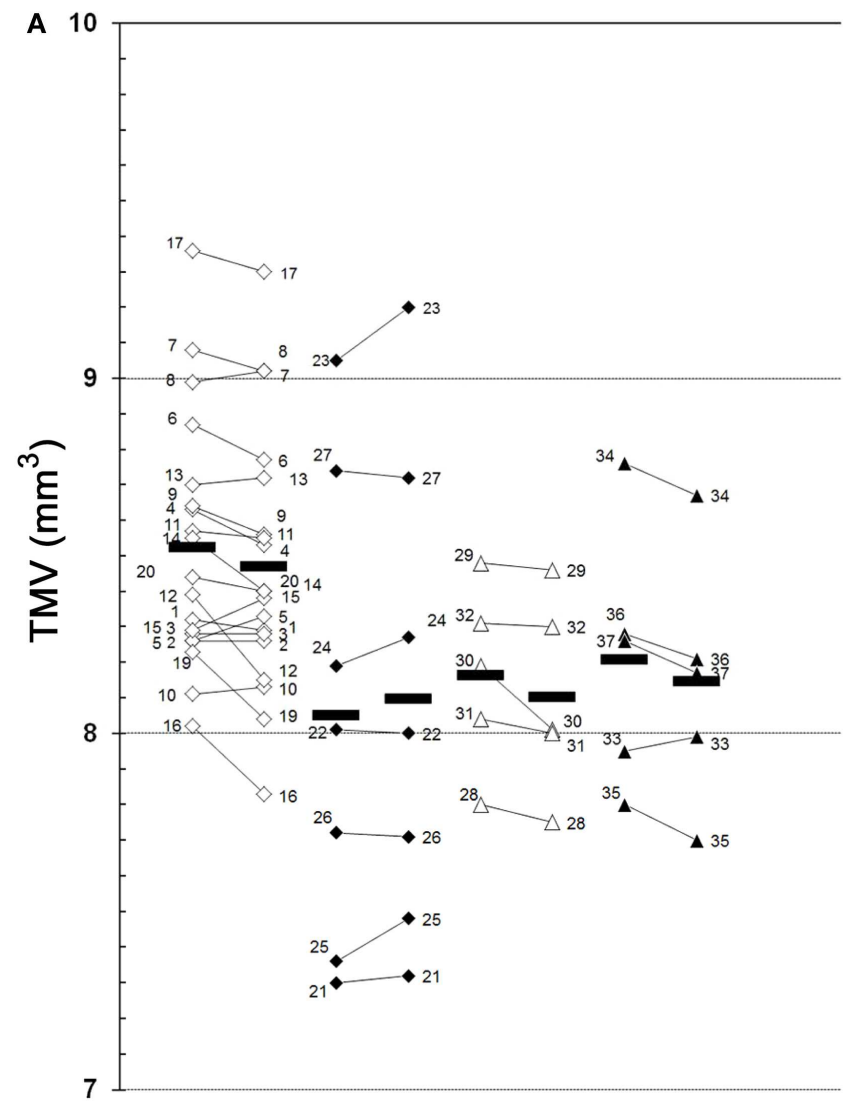

FIGURE 1 | Global TMV changes between baseline and follow-up examination. (A), left eye; (B), right eye; 1-37, patient 1-37 (see Table 1 for demographic and clinical data); white squares, RRMS without ON

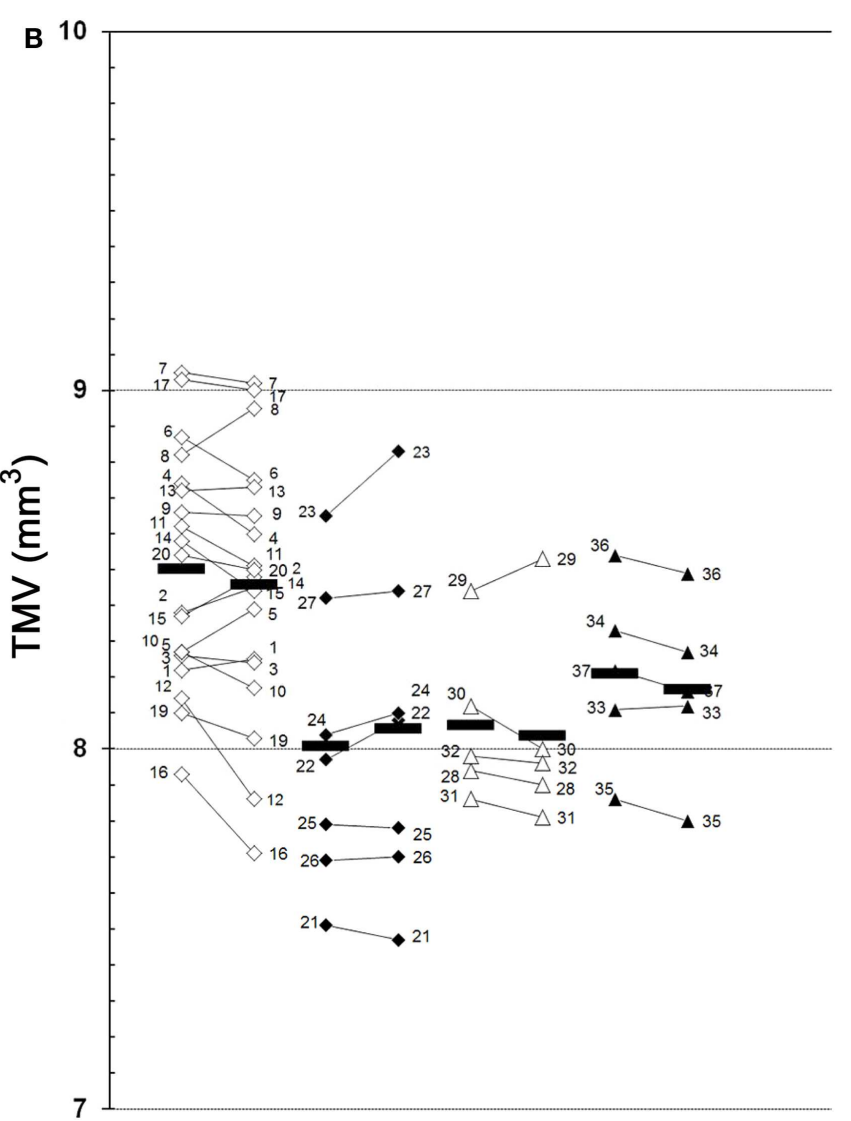

(baseline $\biguplus$ follow-up); black squares, RRMS with ON (baseline $\longmapsto$ follow-up); white triangles, SPMS without ON (baseline 1 follow-up); black triangles, SPMS with ON (baseline $\longmapsto$ follow-up); black bars, means. 

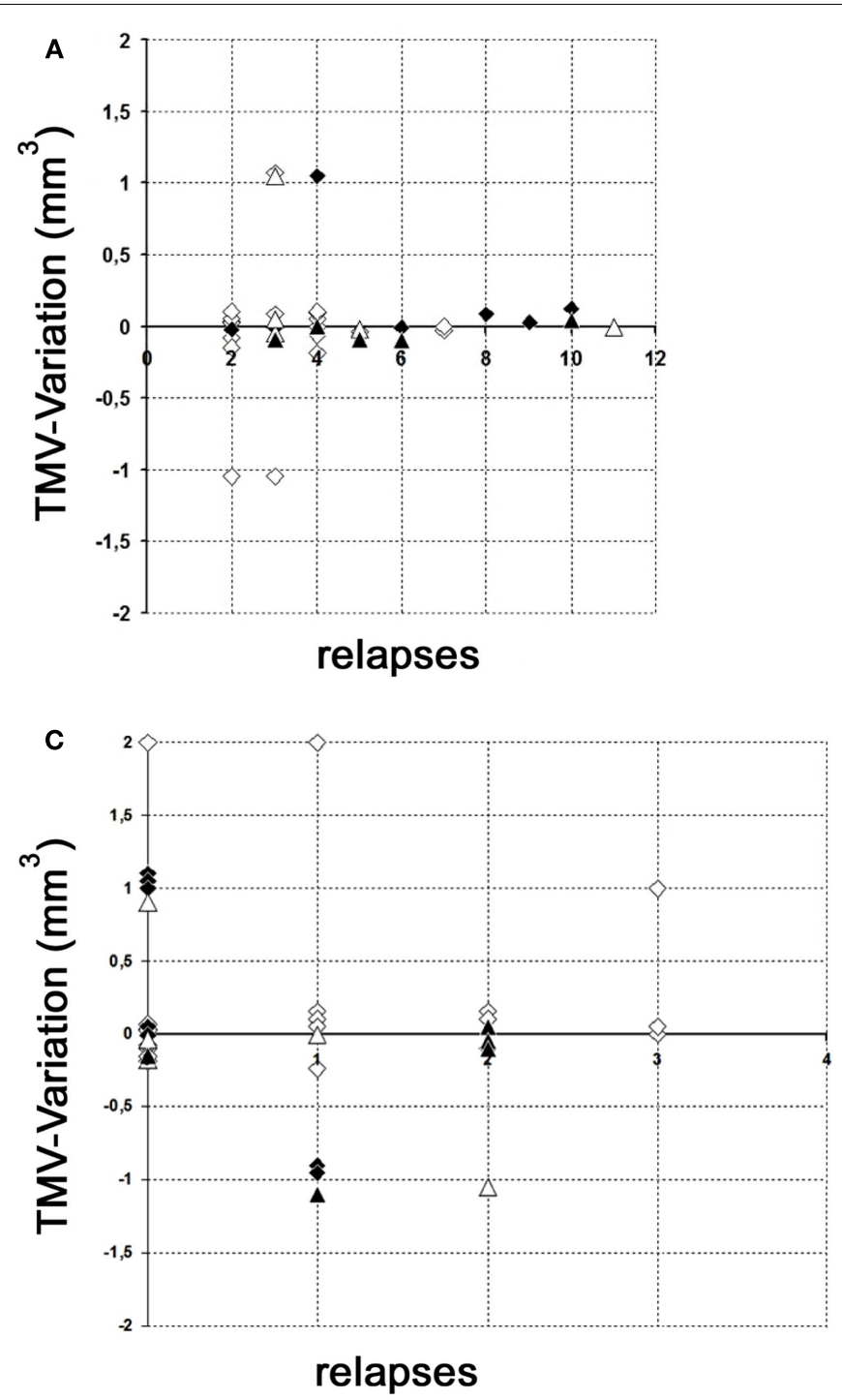

FIGURE 2 | Variation of global TMV measurements. (A,B) Variation of RNFL measurements between baseline and follow-up examination in relation to the total relapses (without ON) before study entry. (C,D) Variation of RNFL

measurements between baseline and follow-up examination in relation to the
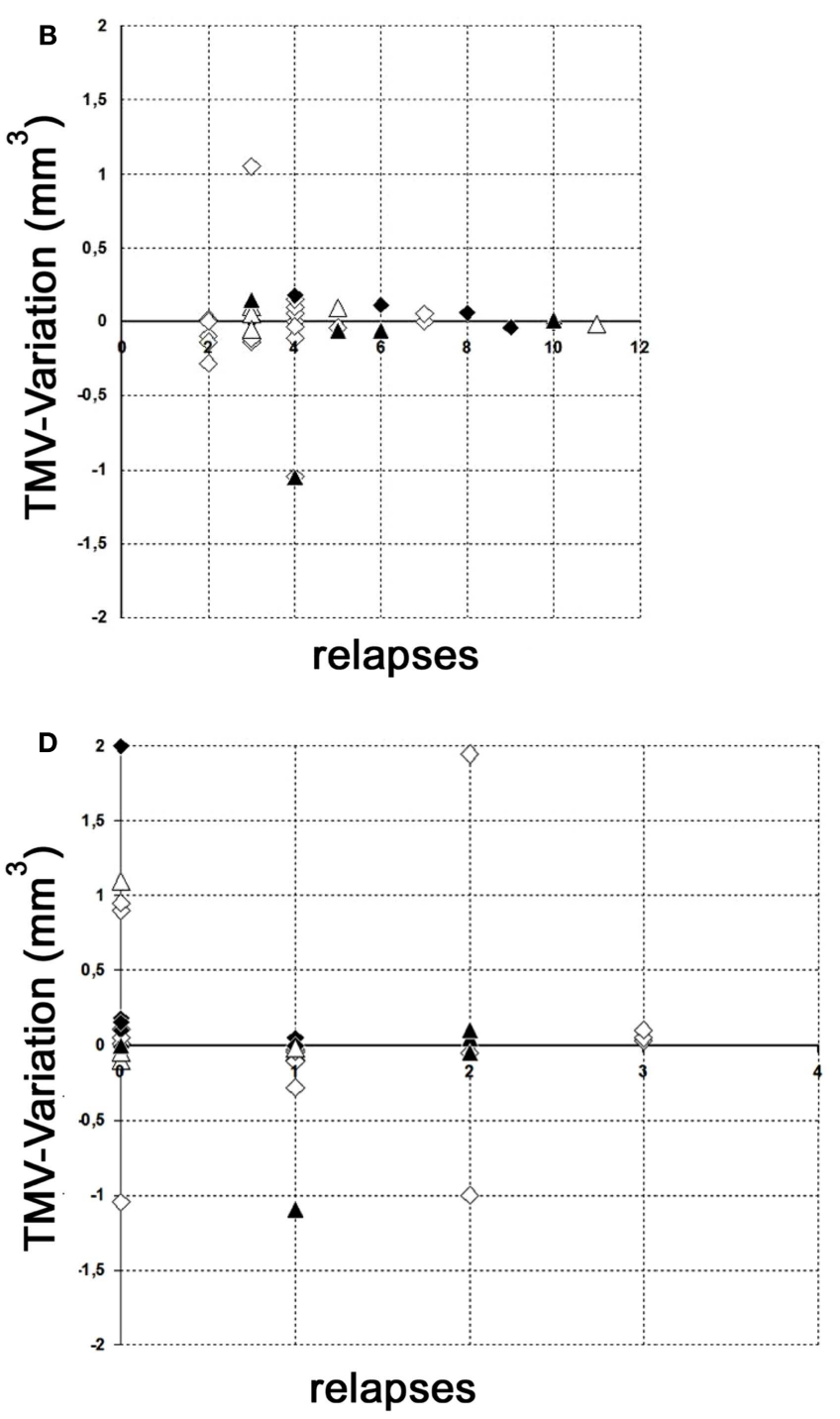

relapses (without ON) during the observation period. (A) Left eye; (B) right eye; (C) left eye; (D) right eye; white squares, RRMS without ON; black squares, RRMS with ON; white triangles, SPMS without ON; black triangles, SPMS with ON.

Nevertheless, (1) large normative databases and (2) longitudinal studies performed with high-resolution SD-OCT devices, large patient numbers and controls (at least several hundred), and long observation periods over several years ( $>5$ years) are lacking. Meta-analyses are limited as (1) the measurements of individual devices are not interchangeable and comparable (9), (2) inclusion criteria of the patients are not clear, or (3) "affected eyes" were separated from their "unaffected fellow eyes," hence the case numbers being doubled (20). Most importantly, physiological variations of the RNFLT or TMV were found to exceed the detection-threshold of SD-OCT devices and the hypothesized subtle retinal changes of $<2-4 \mu \mathrm{m} /$ year (7).

Both the current study and our previous studies $(6,8)$ give rise to a more critical evaluation of OCT as an efficient monitoring 

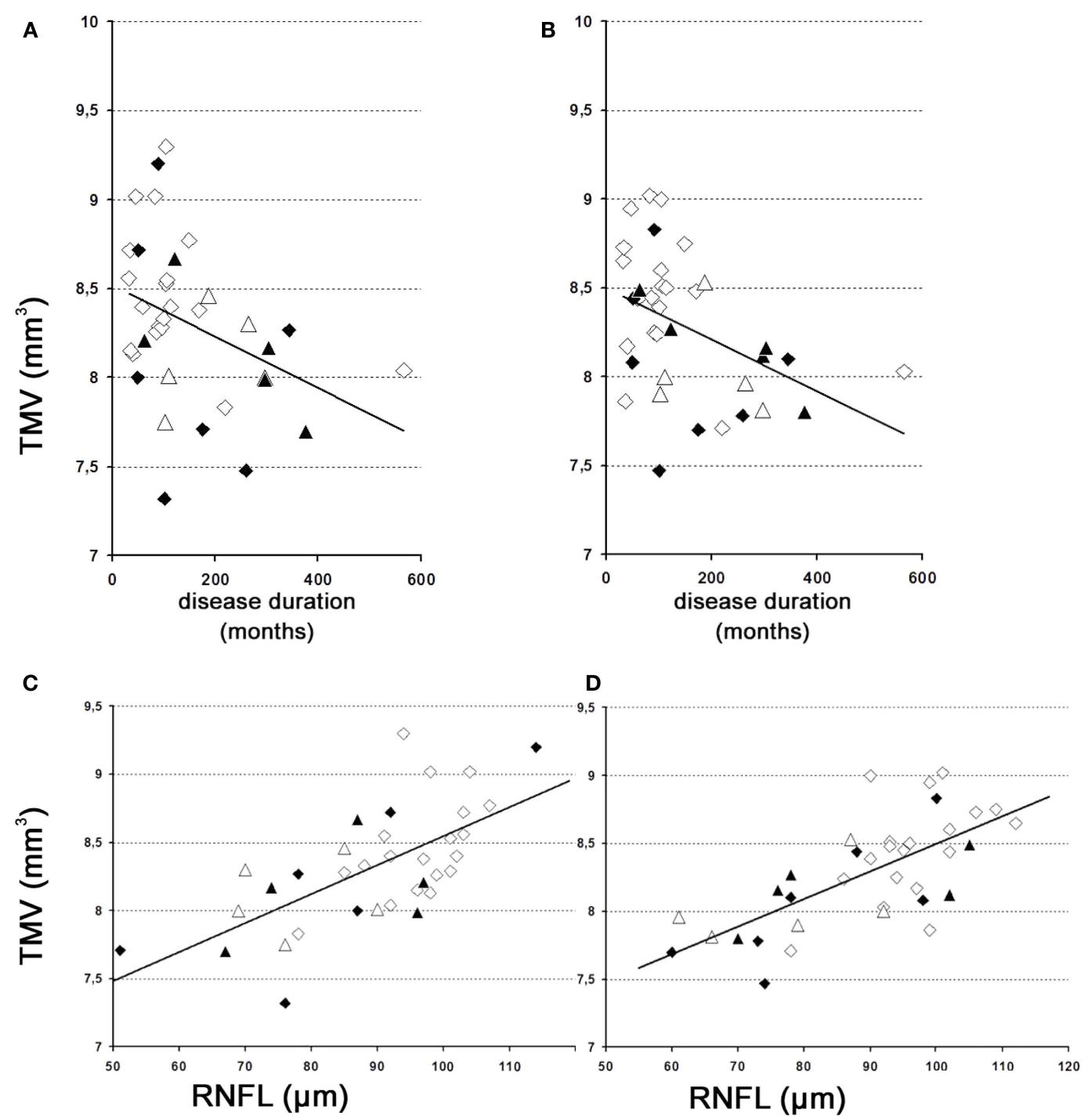

FIGURE 3 |TMV over time. (A,B) Changes of the TMV over time. (A) Left eye - weak correlation of global TMV changes over time. $y=8.5-0.001 \times$ $x$ [correlation coefficient $=-0.4 ; R^{2}=18.7 \%$; standard error of estimate $=$ 0.4]. (B) Right eye - weak correlation of global TMV changes over time. $y=8.5-0.001 \times x$ [correlation coefficient $=-0.4 ; R^{2}=14.4 \%$; standard error of estimate $=0.4]$. (C,D) Correlations between TMV and the RNFL. (C) Left eye - moderately strong relationship between TMV and RNFL. $y=6.4+0.02 \times x$ [correlation coefficient $=0.7 ; R^{2}=47.9 \%$; standard error of estimate $=0.3$. (D) Right eye - moderately strong relationship between TMV and RNFL. $y=6.4+0.02 \times x$ [correlation coefficient $=0.7$; $R^{2}=48.5 \%$; standard error of estimate $\left.=0.3\right]$. (A) Left eye; (B) right eye; (C) left eye; (D), right eye; white squares, RRMS without ON; black squares, RRMS with ON; white triangles, SPMS without ON; black triangles, SPMS with ON. instrument for MS disease progression, and thus any interpretation of therapeutic studies must proceed with the utmost caution $(1-3,6-8,12,18-21)$.

\section{REFERENCES}

1. Warner CV, Syc SB, Stankiewicz AM, Hiremath G, Farrell SK, Crainiceanu CM, et al. The impact of utilizing different optical coherence tomography devices for clinical purposes and in multiple sclerosis trials. PLoS One (2011) 6(8):e22947. doi:10.1371/journal.pone.0022947

2. Dorr J, Wernecke KD, Bock M, Gaede G, Wuerfel JT, Pfueller CF, et al. Association of retinal and macular damage with brain atrophy in multiple sclerosis. PLoS One (2011) 6(4):e18132. doi:10.1371/journal.pone.0018132
3. Burkholder BM, Osborne B, Loguidice MJ, Bisker E, Frohman TC, Conger A, et al. Macular volume determined by optical coherence tomography as a measure of neuronal loss in multiple sclerosis. Arch Neurol (2009) 66(11):1366-72. doi:10.1001/archneurol.2009.230

4. Gilmore CP, Cottrell DA, Scolding NJ, Wingerchuk DM, Weinshenker BG, Boggild $\mathrm{M}$, et al. A window of opportunity for no treatment in early multiple sclerosis? Mult Scler (2010) 16(6):756-9. doi:10.1177/1352458510366014

5. Marburg O. The amygdaloid complex. Confin Neurol (1949) 9(3-4):211-6. doi:10.1159/000105833

6. Serbecic N, Aboul-Enein F, Beutelspacher SC, Graf M, Kircher K, Geitzenauer $\mathrm{W}$, et al. Heterogeneous pattern of retinal nerve fiber layer in multiple sclerosis. High resolution optical coherence tomography: potential and limitations. PLoS One (2010) 5(11):e13877. doi:10.1371/journal.pone.0013877 
7. Balk LJ, Sonder JM, Strijbis EM, Twisk JW, Killestein J, Uitdehaag BM, et al. The physiological variation of the retinal nerve fiber layer thickness and macular volume in humans as assessed by spectral domain-optical coherence tomography. Invest Ophthalmol Vis Sci (2012) 53(3):1251-7. doi:10.1167/iovs.11-8209

8. Serbecic N, Aboul-Enein F, Beutelspacher SC, Vass C, Kristoferitsch W, Lassmann $\mathrm{H}$, et al. High resolution spectral domain optical coherence tomography (SD-OCT) in multiple sclerosis: the first follow up study over two years. PLoS One (2011) 6(5):e19843. doi:10.1371/journal.pone.0019843

9. Wolf-Schnurrbusch UE, Ceklic L, Brinkmann CK, Iliev ME, Frey M, Rothenbuehler SP, et al. Macular thickness measurements in healthy eyes using six different optical coherence tomography instruments. Invest Ophthalmol Vis Sci (2009) 50(7):3432-7. doi:10.1167/iovs.08-2970

10. Aboul-Enein F, Krssak M, Hoftberger R, Prayer D, Kristoferitsch W. Reduced NAA-levels in the NAWM of patients with MS is a feature of progression. A study with quantitative magnetic resonance spectroscopy at 3 Tesla. PLoS One (2010) 5(7):e11625. doi:10.1371/journal.pone.0011625

11. Krampla W, Aboul-Enein F, Jecel J, Lang W, Fertl E, Hruby W, et al. Spinal cord lesions in patients with neuromyelitis optica: a retrospective long-term MRI follow-up study. Eur Radiol (2009) 19(10):2535-43. doi:10.1007/s00330-0091425-3

12. Mader S, Gredler V, Schanda K, Rostasy K, Dujmovic I, Pfaller K, et al. Complement activating antibodies to myelin oligodendrocyte glycoprotein in neuromyelitis optica and related disorders. J Neuroinflammation (2011) 8:184. doi:10.1186/1742-2094-8-184

13. Frohman EM, Fujimoto JG, Frohman TC, Calabresi PA, Cutter G, Balcer LJ. Optical coherence tomography: a window into the mechanisms of multiple sclerosis. Nat Clin Pract Neurol (2008) 4(12):664-75. doi:10.1038/ncpneuro0950

14. Henderson AP, Trip SA, Schlottmann PG, Altmann DR, Garway-Heath DF, Plant GT, et al. An investigation of the retinal nerve fibre layer in progressive multiple sclerosis using optical coherence tomography. Brain (2008) 131(Pt 1):277-87. doi:10.1093/brain/awm285

15. Petzold A, de Boer JF, Schippling S, Vermersch P, Kardon R, Green A, et al. Optical coherence tomography in multiple sclerosis: a systematic review and meta-analysis. Lancet Neurol (2010) 9(9):921-32. doi:10.1016/S1474-4422(10) 70168-X

16. Henderson AP, Altmann DR, Trip AS, Kallis C, Jones SJ, Schlottmann PG, et al. A serial study of retinal changes following optic neuritis with sample size estimates for acute neuroprotection trials. Brain (2010) 133(9):2592-602. doi:10.1093/brain/awq146
17. Serbecic N, Beutelspacher SC, Aboul-Enein FC, Kircher K, Reitner A, SchmidtErfurth U. Reproducibility of high-resolution optical coherence tomography measurements of the nerve fibre layer with the new Heidelberg Spectralis optical coherence tomography. Br J Ophthalmol (2011) 95(6):804-10. doi:10.1136/bjo.2010.186221

18. Dinkin M, Paul F. Higher macular volume in patients with MS receiving fingolimod: positive outcome or side effect? Neurology (2013) 80(2):128-9. doi:10.1212/WNL.0b013e31827ccf4a

19. Nolan R, Gelfand JM, Green AJ. Fingolimod treatment in multiple sclerosis leads to increased macular volume. Neurology (2013) 80(2):139-44. doi:10.1212/WNL.0b013e31827b9132

20. Talman LS, Bisker ER, Sackel DJ, Long DAJr, Galetta KM, Ratchford JN, et al. Longitudinal study of vision and retinal nerve fiber layer thickness in multiple sclerosis. Ann Neurol (2010) 67(6):749-60. doi:10.1002/ana.22005

21. Henderson AP, Trip SA, Schlottmann PG, Altmann DR, Garway-Heath DF, Plant GT, et al. A preliminary longitudinal study of the retinal nerve fiber layer in progressive multiple sclerosis. J Neurol (2010) 257(7):1083-91. doi:10.1007/s00415010-5467-x

Conflict of Interest Statement: The authors declare that the research was conducted in the absence of any commercial or financial relationships that could be construed as a potential conflict of interest.

Received: 23 June 2013; accepted: 07 February 2014; published online: 24 February 2014.

Citation: Serbecic N, Aboul-Enein F, Beutelspacher SC, Khan A, Vass C, Kristoferitsch $W$, Reitner A and Schmidt-Erfurth U (2014) High-resolution spectral domain-optical coherence tomography in multiple sclerosis, Part II - the total macular volume. The first follow-up study over 2 years. Front. Neurol. 5:20. doi: 10.3389/fneur.2014.00020

This article was submitted to Neuro-Ophthalmology, a section of the journal Frontiers in Neurology.

Copyright (C) 2014 Serbecic, Aboul-Enein, Beutelspacher, Khan, Vass, Kristoferitsch, Reitner and Schmidt-Erfurth. This is an open-access article distributed under the terms of the Creative Commons Attribution License (CC BY). The use, distribution or reproduction in other forums is permitted, provided the original author(s) or licensor are credited and that the original publication in this journal is cited, in accordance with accepted academic practice. No use, distribution or reproduction is permitted which does not comply with these terms. 